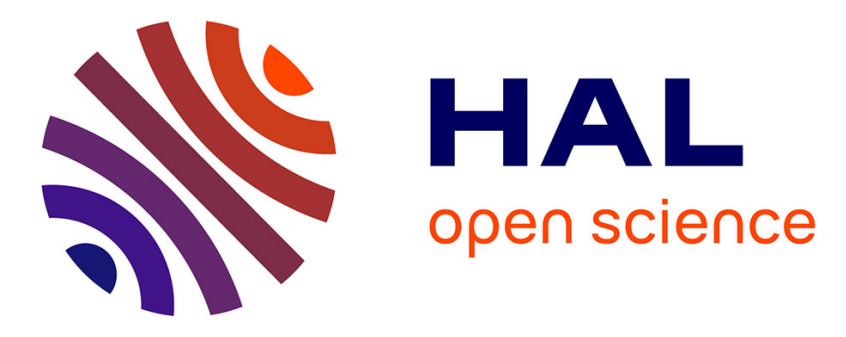

\title{
Enhancing the linear dynamic range of a mode-localized MEMS mass sensor with repulsive electrostatic actuation
}

\author{
Toky Harrison Rabenimanana, Vincent Walter, Najib Kacem, Patrice Le \\ Moal, Gilles Bourbon, Joseph Lardies
}

\section{To cite this version:}

Toky Harrison Rabenimanana, Vincent Walter, Najib Kacem, Patrice Le Moal, Gilles Bourbon, et al.. Enhancing the linear dynamic range of a mode-localized MEMS mass sensor with repulsive electrostatic actuation. Smart Materials and Structures, 2021, 30 (7), pp.07LT01 (6). hal-03421028

\section{HAL Id: hal-03421028 \\ https://hal.science/hal-03421028}

Submitted on 9 Nov 2021

HAL is a multi-disciplinary open access archive for the deposit and dissemination of scientific research documents, whether they are published or not. The documents may come from teaching and research institutions in France or abroad, or from public or private research centers.
L'archive ouverte pluridisciplinaire HAL, est destinée au dépôt et à la diffusion de documents scientifiques de niveau recherche, publiés ou non, émanant des établissements d'enseignement et de recherche français ou étrangers, des laboratoires publics ou privés. 


\title{
Enhancing the linear dynamic range of a mode-localized MEMS mass sensor with repulsive electrostatic actuation
}

\author{
T Rabenimanana, V Walter, N Kacem, P Le Moal, G \\ Bourbon and $\mathbf{J}$ Lardiès \\ Univ. Bourgogne Franche-Comté, FEMTO-ST Institute, \\ CNRS/UFC/ENSMM/UTBM, Department of Applied Mechanics, 25000 \\ Besançon, France \\ E-mail: toky.rabenimanana@femto-st.fr \\ March 2021
}

\begin{abstract}
This letter demonstrates the linear dynamic range enhancement of a mode-localized MEMS sensor based on two weakly coupled cantilevers under electrostatic actuation resulting in a repulsive force. An analytical model is proposed to design the sensor, and the expression of the electrostatic force is obtained using FEM simulation. Compared to attractive electrostatic actuation, the intensity of the resulting force is less sensitive to the change in the cantilever's displacement, with negligible electrostatic nonlinearities. This result is confirmed by experimental measurements showing linear vibrations up to $70 \%$ of the gap, which is almost three times higher than the electrostatic critical amplitude of a similar device using attractive electrostatic force. Finally, the mass sensing capability is highlighted by depositing a few picograms of platinum on the sensor.
\end{abstract}

Keywords: Mode localization, mass sensor, linear dynamic range, repulsive force, electrostatic actuation 


\section{Introduction}

MEMS mass sensors exploiting the mode localization [1] have been considered in several studies over the last decade. Compared to sensors using the frequency shift of a single resonator, this new generation of sensor is less sensitive to the ambient condition changes [2]. To detect a mass, it measures the change in the vibration mode of a system composed of weakly coupled identical resonators. The ability to measure a mass with this principle has been proposed for the first time in [3] with a device composed of two mechanically coupled cantilevers. Then, other studies proposing different types of mode-localized sensors with various applications have emerged [4]. It also concerns other applications like energy harvesting $[5,6]$. For MEMS sensors, the authors often investigate the number of coupled resonators [7-9], and the use of other kind of resonators and coupling [10,11]. Recently, the coupling between resonators of different physical nature has been proposed $[12,13]$.

To determine the added mass on mode-localized sensors, there are many output metrics that can be considered [14], but they are all based on vibration amplitude measurement. In order to improve the mass resolution of the sensor, one should be able to drive the resonators at high vibration amplitude. Besides, it is shown in [15] that the resolution is inversely dependent on the driving force for sensor based on coupled linear resonators. Consequently, the upper bound limit of the dynamic range of the used resonator should be enhanced.

For mode-localized sensors using cantilevers actuated with electrostatic attractive force, the linear dynamic range is limited by the critical amplitude [16] often imposed by electrostatic nonlinearities. Above this critical amplitude, the resonator shows a multi-valued response. Our previous work [17] shows the advantage of driving such a device beyond this limit, i.e. involving nonlinear behavior, but there is still the risk of failure due to the pull-in phenomenon. However, there is another type of electrostatic actuation which is based on repulsive force. It is often used for micromirrors $[18,19]$, but it can also be used for cantilevers [20,21]. This actuation has the advantage of achieving large stroke [22] while avoiding pull-in instability. While mode-localized sensors with attractive electrostatic forces have been widely studied, to the best of our knowledge, the implementation of repulsive forces for the actuation of such sensors has not been investigated. Thus, we propose in this letter to study theoretically and experimentally the use of this type of electrostatic actuation with mode-localized sensors composed of weakly coupled MEMS cantilevers. We first present the design and the modeling of the proposed device. Then, the experimental study is carried out with the fabricated device.

\section{Design and model}

As shown in Figure 1, The sensor considered in this study is composed of two cantilevers connected by a coupling beam near the fixed end. Under each cantilever, we have a bottom electrode with two side electrodes. To generate the vibration of the system, we actuate one of the two cantilevers (Cantilever 1) by applying a combined AC-DC voltage on its two side electrodes, while the two cantilevers, the bottom electrodes, and the two side electrodes around the nonactuated cantilever (Cantilever 2) are grounded. The

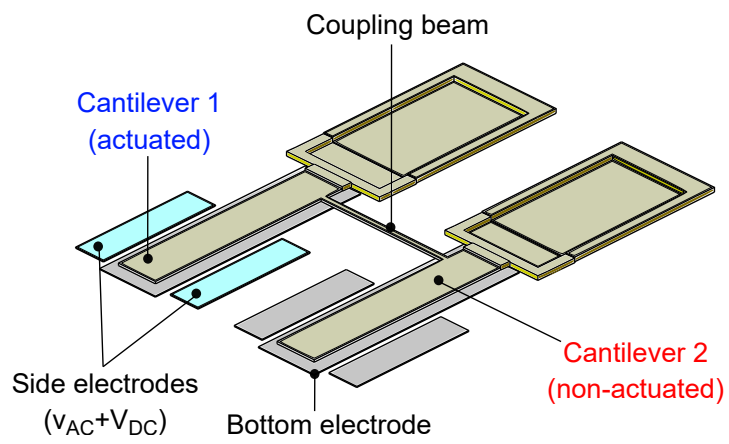

Figure 1. The two coupled cantilevers using repulsive electrostatic actuation.

dimensions of the device are given in Table 1 where the gap $g$ represents the distance between the cantilever and the bottom electrode.

Table 1. Dimensions of the device

\begin{tabular}{ll}
\hline Dimension & Value \\
\hline Length of Cantilever 1 & $L_{1}=100 \mu \mathrm{m}$ \\
Length of Cantilever 2 & $L_{2}=100 \mu \mathrm{m}$ \\
Width of the cantilevers & $b=20 \mu \mathrm{m}$ \\
Thickness of the cantilevers & $h=1.25 \mu \mathrm{m}$ \\
Length of the coupling beam & $L_{c}=65 \mu \mathrm{m}$ \\
Width of the coupling beam & $b_{c}=3 \mu \mathrm{m}$ \\
Position of the coupling beam & $\tilde{x}_{c}=4.6 \mu \mathrm{m}$ \\
Gap & $g=1 \mu \mathrm{m}$ \\
Width of the bottom electrode & $b_{b}=28 \mu \mathrm{m}$ \\
Width of the side electrodes & $b_{s e}=15 \mu \mathrm{m}$ \\
Position of the side electrodes & $\tilde{x}_{e}=40 \mu \mathrm{m}$ \\
Distance between each electrode & $g_{e}=3 \mu \mathrm{m}$ \\
\hline
\end{tabular}

Before modeling the device, we first determine the expression of the electrostatic force applied to Cantilever 1. An analytical model of the force has already been proposed in [23] for this kind of actuation. But for greater accuracy, we choose to perform a simulation with COMSOL Multiphysics ${ }^{\circledR}$. To do so, we consider a 2D FEM model (Figure 2(a)) composed of the cross sections of the two cantilevers, the bottom 
electrodes, the side electrodes, and the surrounding medium. We then apply a DC voltage of $1 \mathrm{~V}$ on the two side electrodes of Cantilever 1, and the other electrodes and the two cantilevers are grounded. The mechanical displacement of the cantilever is blocked in order to determine the electrostatic force exerted by measuring the reaction force on the upper side of the cantilever. After calculation, the electric potential and electric field lines are shown in Figure 2(b). We then perform simulations with different values of the distance between the cantilever and the bottom electrode $\left(g+\tilde{w}_{i}\right.$, where $\tilde{w}_{i}$ is the displacement of Cantilever i). Distances between $0.3 \mu \mathrm{m}$ to $3.8 \mu \mathrm{m}$ are considered, and the intensity of the electrostatic force is determined for each of them. The results are shown in Figure 2(c) and (d).

In Figure 2(c), we notice that for the chosen range of the distances, the relative variation of the force intensity for Cantilever 1 is less than $10 \%$. Unlike the attractive electrostatic force which is inversely proportional to the square of $g+\tilde{w}_{1}$, the repulsive force is less dependent on the cantilever displacement. Consequently, the softening or the hardening effects of this actuation are not important. But another consequence is that the electrostatic nonlinearities are negligible. To determine now the expression of the force, we use Matlab R2016b to find the coefficients of the third order polynomial that is the best fit for the curve obtained in Figure 2(c). From this, the intensity of the repulsive electrostatic force $\tilde{F}_{E}$ per unit length of Cantilever 1 and for a voltage of $1 V$ is given by

$$
\begin{aligned}
\tilde{F}_{E}=\tilde{C}_{0}+\tilde{C}_{1} & \left(g+\tilde{w}_{1}\right) \\
& +\tilde{C}_{2}\left(g+\tilde{w}_{1}\right)^{2}+\tilde{C}_{3}\left(g+\tilde{w}_{1}\right)^{3}
\end{aligned}
$$

where $\tilde{C}_{0}=1.8010^{-7} \mathrm{~N} / \mathrm{m} / V^{2}, \quad \tilde{C}_{1}=$ $2.8410^{-2} \mathrm{~N} / \mathrm{m}^{2} / \mathrm{V}^{2}, \quad \tilde{C}_{2}=-1.1610^{4} \mathrm{~N} / \mathrm{m}^{3} / V^{2}$ and $\tilde{C}_{3}=8.7010^{8} \mathrm{~N} / \mathrm{m}^{4} / \mathrm{V}^{2}$. For other voltages, this expression is just multiplied by $V^{2}$ (where $V$ is the voltage used). Concerning the non-actuated cantilever, the simulation result in Figure 2(d) shows that there is also a small force applied to Cantilever 2 , and its intensity is approximately equal to $3 \%$ of that of the force applied to Cantilever 1 . To consider this force in the analytical model, we assume that for Cantilever 2, the electrostatic force intensity per unit length is equal to $0.03 \tilde{F}_{E}$.

Once we get the expression of the electrostatic force, we can study the dynamic behavior of the device. As the proposed device presents similarities with that studied in our previous work [24], we use for the mechanical part the same analytical model which is composed of two Euler- Bernoulli beams connected by a torsional spring. So, the equation governing the (a)

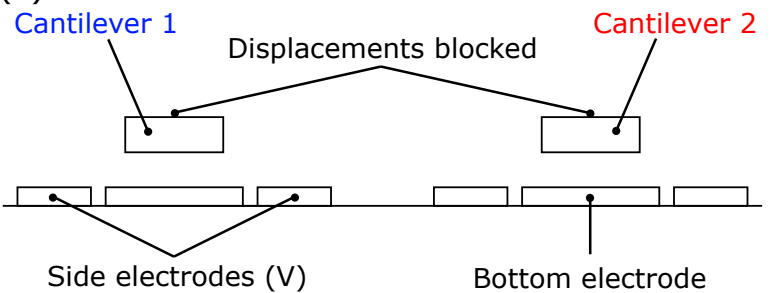

(b)
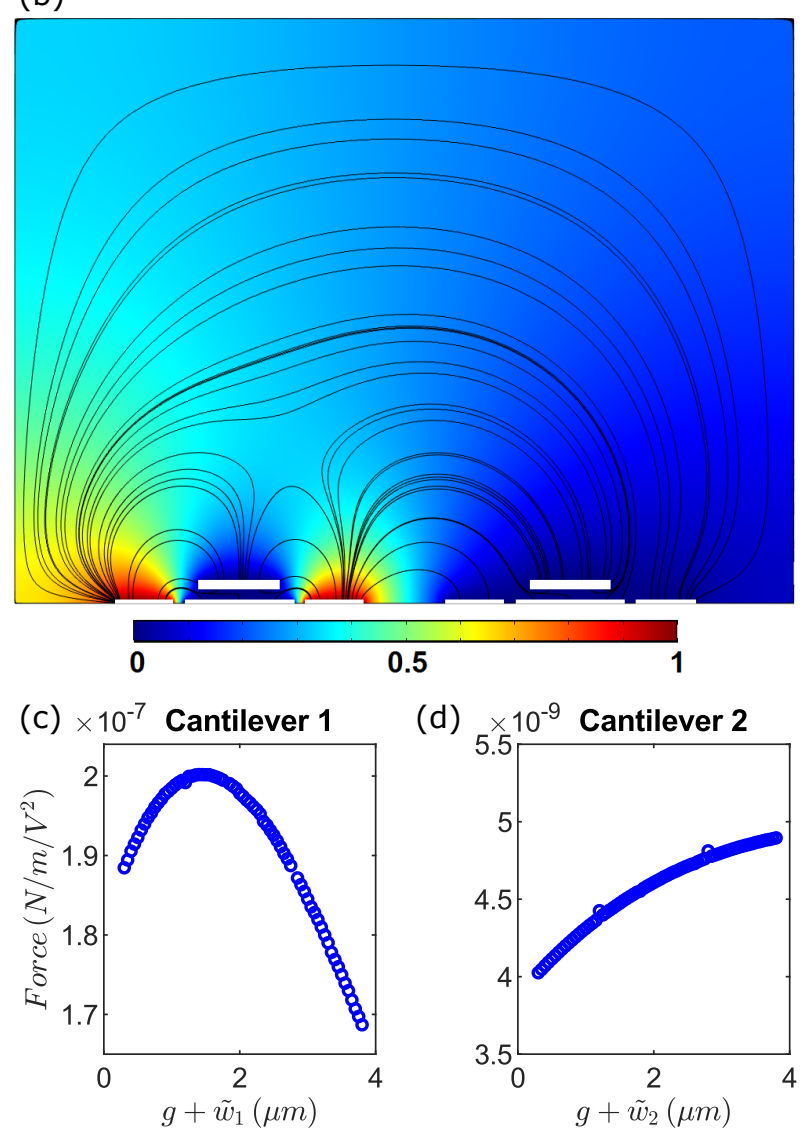

(d) $\times 10^{-9}$ Cantilever 2

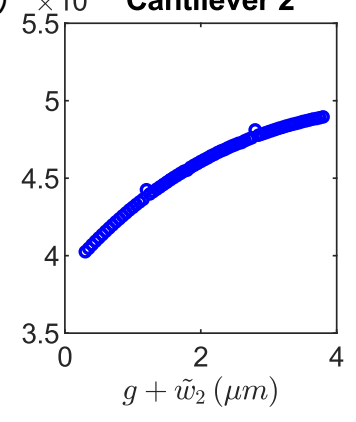

Figure 2. (a) Schematic of the 2D FEM model, (b) electric potential and electric field lines for a given value of the distance between the cantilever and the bottom electrode, (c) and (d) intensity of the electrostatic force on Cantilever 1 and Cantilever 2 as a function of the distance between the cantilever and the bottom electrode.

vibration of the system is given by

$$
\begin{aligned}
& E I \tilde{w}_{1}^{\prime \prime \prime \prime}+\left(\rho b h+\tilde{m} \delta\left(\tilde{x}-\tilde{x}_{m}\right)\right) \ddot{\tilde{w}}_{1}+\tilde{c}_{1} \dot{\tilde{w}}_{1} \\
& \quad-\tilde{k}_{r}\left(\tilde{w}_{1}^{\prime}\left(\tilde{x}_{c}\right)-\tilde{w}_{2}^{\prime}\left(\tilde{x}_{c}\right)\right) \delta^{\prime}\left(\tilde{x}-\tilde{x}_{c}\right) \\
& \quad=H\left(\tilde{x}-\tilde{x}_{e}\right)\left(V_{D C}+v_{A C} \cos (\tilde{\Omega} \tilde{t})\right)^{2} * \tilde{F}_{E}
\end{aligned}
$$

$$
\begin{aligned}
& E I \tilde{w}_{2}^{\prime \prime \prime \prime}+\rho b h \ddot{\tilde{w}}_{2}+\tilde{c}_{2} \dot{\tilde{w}}_{2} \\
& \quad-\tilde{k}_{r}\left(\tilde{w}_{2}^{\prime}\left(\tilde{x}_{c}\right)-\tilde{w}_{1}^{\prime}\left(\tilde{x}_{c}\right)\right) \delta^{\prime}\left(\tilde{x}-\tilde{x}_{c}\right) \\
& \quad=H\left(\tilde{x}-\tilde{x}_{e}\right)\left(V_{D C}+v_{A C} \cos (\tilde{\Omega} \tilde{t})\right)^{2} * 0.03 \tilde{F}_{E}
\end{aligned}
$$

where primes and dots denote respectively the partial differentiation with respect to the position along the 
microbeam $\tilde{x}$ and to the time $\tilde{t}, E$ is the Young's modulus of the constitutive material, $I$ is the moment of inertia of the cross section, $\tilde{w}_{i}(i=1,2)$ is the deflection of Cantilever $i, \rho$ is the material density, $m$ is the mass perturbation, $\tilde{x}_{m}$ is its position, $\tilde{c}_{i}$ is the damping coefficient, $\tilde{k}_{r}$ is the torsional stiffness of the coupling beam, $\delta$ is the Dirac delta function, $H$ is the Heaviside function, and $v_{A C}$ and $V_{D C}$ are respectively the AC and DC actuation voltages. To solve (2) and (3), we use one mode Galerkin discretization, and readers can see supplementary material for more details. Since the electrostatic force is less dependent on the displacement, we have neglected the quadratic and cubic nonlinearities in the final equation.

One of the problems encountered on modelocalized sensors is the microfabrication tolerance that prevents us from having perfectly identical coupled resonators. To counterbalance the effect of these manufacturing defects, a previous work [25] proposes to use an asymmetrical device where the short microbeam which is actuated is $98 \mu \mathrm{m}$ long while the other one is $100 \mu \mathrm{m}$ long. The softening effect of the attractive electrostatic actuation is then used by tuning the DC voltage in order to reduce the effective stiffness of the short cantilever, thus allowing to balance the system. In our case, the theoretical results in Figure 3 show that the electrostatic softening effect is much less present with repulsive electrostatic forces. As a result, an identical length of $100 \mu \mathrm{m}$ was chosen for both cantilevers on the layout, and the DC voltage will only be used to compensate for the asymmetry created by the manufacturing defects.

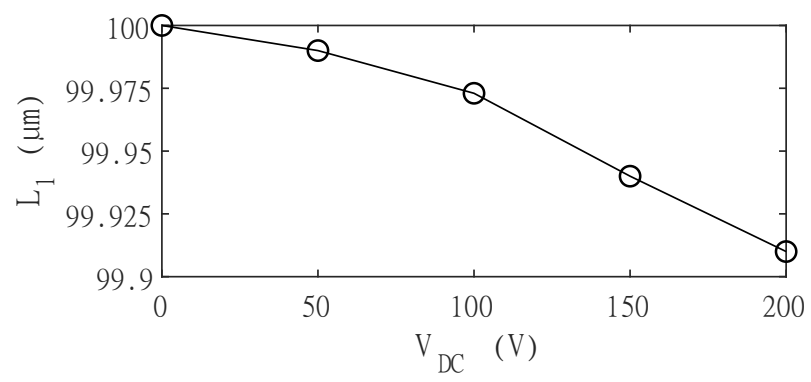

Figure 3. Length of the actuated cantilever as a function of the required DC voltage (the length of the non actuated cantilever is equal to $100 \mu \mathrm{m})$.

\section{Experimental results}

After the modeling and the design, we use the PolyMUMPS ${ }^{\circledR}$ process [26] to fabricate the device. It is then placed in a vacuum chamber, and the vibration at the free end of each cantilever is measured with a single point laser Doppler vibrometer (LDV). As shown in Figure 4, the experimental measurement is performed with a Picoscope ${ }^{\circledR} 5444 \mathrm{~B}$ that supplies the actuation voltage, and measures the signal from the LDV. To balance the device, we have to actuate

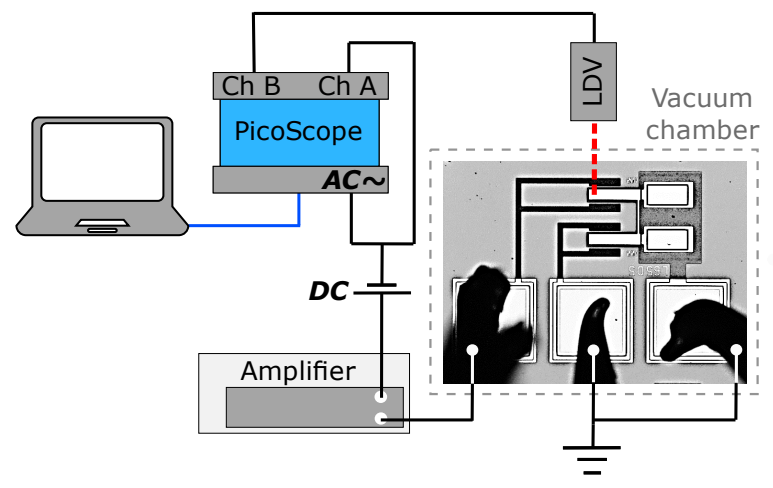

Figure 4. Schematic of the experimental setup.

the cantilever having the highest effective stiffness. To determine this, we actuate simultaneously both cantilevers. The microbeam with the lowest stiffness will then vibrate more in the first mode (at the lowest frequency), while the one with the highest stiffness will vibrate more in the second mode. Once the cantilever to be actuated is identified, we apply the combined AC-DC voltage to its side electrodes. We then increase gradually the DC voltage to decrease its effective stiffness, until the vibration amplitudes of the cantilevers become identical on the two modes (symmetric mode and antisymmetric mode). For the chosen device, a DC voltage $V_{D C}=110 \mathrm{~V}$ was required to reach the balanced state. We then perform simulation with this same voltage for the theoretical frequency response. A quality factor $Q=900$ is used, and it is measured from the half-power bandwidth method. The identification of the balancing DC voltage of $110 \mathrm{~V}$ proves that there is a slight difference between the two manufactured cantilevers. Thus, in the model, we have to include this defect by decreasing slightly the length of the supposed actuated cantilever so that the balanced state is reached with this same DC voltage of $110 \mathrm{~V}$. Therefore, we set $L_{1}=99.96 \mu \mathrm{m}$ in the model, while $L_{2}$ is kept at $100 \mu m$ (according to Figure 3). The theoretical and experimental frequency responses at the balanced state are shown in Figure 5, where $W_{1 t h} / W_{1 \exp }$ and $W_{2 t h} / W_{2 \exp }$ are respectively the theoretical/experimental vibration amplitude at the end of the actuated and the non-actuated cantilevers.

The comparison between the experimental and theoretical frequency responses shows that they are in good agreement, which allows us to validate the model. We also notice that for $V_{D C}=110 \mathrm{~V}$, the vibration amplitude of each cantilever are identical on each mode, reflecting the balanced state. For other tested devices, the required DC voltages to obtain this balanced state do not exceed $110 \mathrm{~V}$. Concerning the theoretical amplitude of each mode (Figure 5(a)), that of the first 

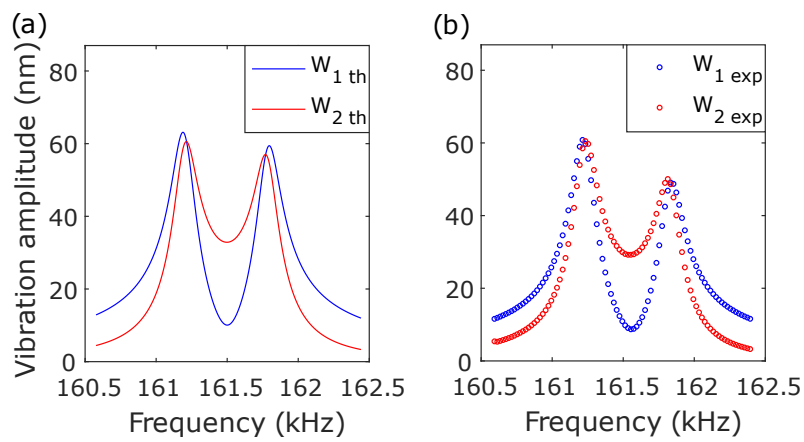

Figure 5. Frequency responses of the device at the balanced state for $v_{A C}=125 \mathrm{mV}$ and $V_{D C}=110 \mathrm{~V}$ : (a) theoretical frequency response (analytical model) and (b) experimental frequency response.

mode is slightly higher than that of the second mode, due to the parasitic force on Cantilever 2. For the experimental frequency response (Figure 5(b)), the difference between the amplitude of each mode is more significant, which means that the actual parasitic force applied to the non-actuated cantilever is more important. This is due to a representativeness problem of the $2 \mathrm{D}$ simulations of the device. But using the $2 \mathrm{D}$ finite elements model is justified since it is not necessary to determine with a great accuracy this parasitic force for the targeted application which is the mass sensing.

Concerning the linear dynamic range, we now drive the device at high vibration amplitude by increasing the $\mathrm{AC}$ voltage, and the result is shown in Figure 6(a). We notice in this experimental frequency response that the vibration is still linear, we do not have a softening behavior reflecting electrostatic nonlinearities even if the vibration amplitudes are close to $500 \mathrm{~nm}$. To determine the linear range, we plot the variation of the amplitude of the first mode as a function of the applied AC voltage. The result in Figure 6(b) shows that the linear range goes up to about $700 \mathrm{~nm}$ that represents $70 \%$ of the gap. After this limit, the vibration is no longer linear, and the ratio between the amplitude and the $\mathrm{AC}$ voltage decreases. The possible cause of the nonlinearity can be the increase in the damping force, because nonlinearity can be neither electrostatic nor mechanical since the critical mechanical amplitude beyond which mechanical nonlinearity appears, indicates a value of $A_{c m}=6.3 L_{1} / \sqrt{Q}=21 \mu \mathrm{m}[27]$. But compared to a similar device using attractive electrostatic force [17] and where nonlinear vibrations appear as soon as the amplitude exceeds $20 \%$ of the gap, it can be concluded that the linear dynamic range has been increased with the repulsive electrostatic force.

To demonstrate the mass sensing with the sensor, we add a small mass estimated to be around $10 \mathrm{pg}$ (a)

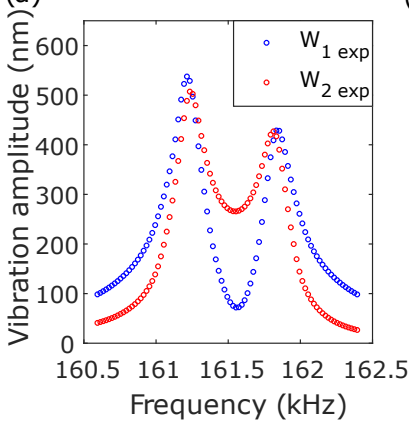

(b)

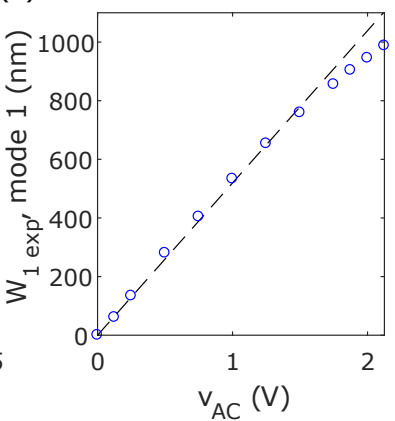

Figure 6. (a) Experimental frequency response of the device with high vibration amplitude $\left(v_{A C}=1 \mathrm{~V}\right.$ and $\left.V_{D C}=110 \mathrm{~V}\right)$ and (b) vibration amplitude of the symmetric mode as a function of the applied $\mathrm{AC}$ voltage

at the free end of the actuated cantilever by using a focused ion beam deposition of platinum (Figure 7(a)). Once the mass is deposited, we measure again the frequency response of the device. We also perform simulation by adding the same mass. All results are shown in Figure 7(b) and (c).

(a)

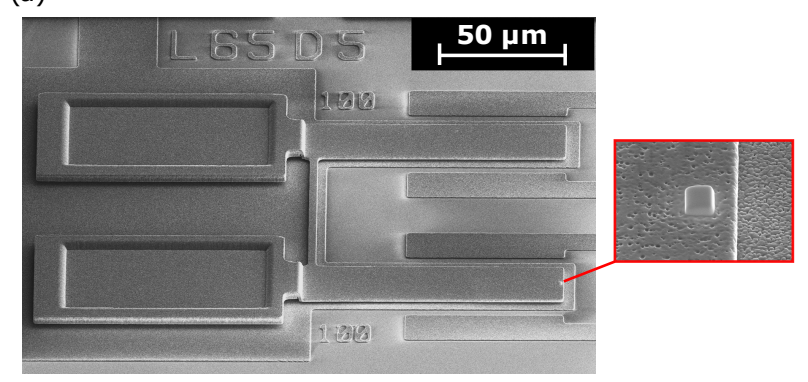

(b)

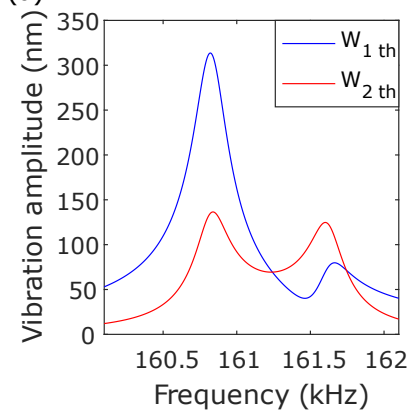

(c)

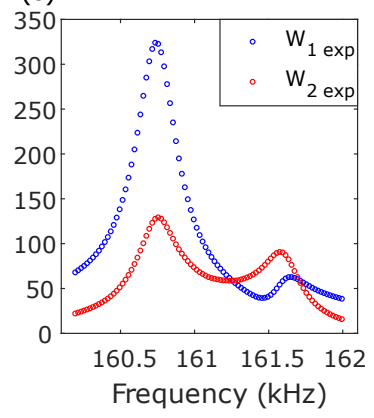

Figure 7. (a) Scanning electron microscope image of the device and the added mass, (b)theoretical and (c) experimental frequency responses of the device once a mass is added on the actuated cantilever $\left(v_{A C}=500 \mathrm{mV}\right.$ and $\left.V_{D C}=110 \mathrm{~V}\right)$

The comparison between theoretical and experimental results shows that they are in good agreement. After adding the mass, the vibration becomes localized on the actuated cantilever in the first mode. When we use high vibration amplitude, we should ensure that the maximum amplitude of this cantilever remains within the linear range described in Figure 6(b). 


\section{Conclusion}

This paper has investigated the use of repulsive electrostatic actuation on a mass sensor with weakly coupled MEMS cantilevers using mode localization. Simulations with a 2D FEM model is first performed to determine the expression of the electrostatic force. The obtained expression is then used in the analytical model based on Euler-Bernoulli beam theory. It shows a resulting force which is less dependent on the displacement of the cantilever than in the case of the attractive electrostatic force, indicating the absence of electrostatic nonlinearities. After carrying out experimentation with the fabricated device, we have also obtained the same results, with a linear range of vibration amplitude up to $70 \%$ of the gap, while the critical amplitude of a similar device using attractive force does not exceed $20 \%$ of the gap. By using a repulsive electrostatic actuation, we have thus increased the upper bound limit of the linear dynamic range of the device. The ability of the sensor to detect a mass of a few picograms is also demonstrated both theoretically and experimentally. Concerning the experimentations, a vibrometer has been used to measure the vibration of the device. But as this may not be relevant in the context of a sensor application, we may investigate in future works the use of other readout techniques that are suitable for nomadic sensors.

\section{Acknowledgments}

This project was supported by the BourgogneFranche-Comté region, the EUR EIPHI program (No. ANR 17-EURE-0002), the French RENATECH network and its FEMTO-ST technological facility

\section{References}

[1] Anderson P W 1958 Physical review 1091492

[2] Thiruvenkatanathan P, Yan J and Seshia A A 2009 Common mode rejection in electrically coupled mems resonators utilizing mode localization for sensor applications 2009 IEEE international frequency control symposium joint with the 22nd European frequency and time forum (IEEE) pp 358-363

[3] Spletzer M, Raman A, Wu A Q, Xu X and Reifenberger R 2006 Applied Physics Letters 88254102
[4] Zhao C, Montaseri M H, Wood G S, Pu S H, Seshia A A and Kraft M 2016 Sensors and Actuators A: Physical 249 93-111

[5] Zergoune Z, Kacem N and Bouhaddi N 2019 Smart Materials and Structures 2807 LT02

[6] Aouali K, Kacem N, Bouhaddi N, Mrabet E and Haddar M 2020 Smart Materials and Structures 29 10LT01

[7] Spletzer M, Raman A, Sumali H and Sullivan J P 2008 Applied Physics Letters 92114102

[8] Wang D F, Li X, Yang X, Ikehara T and Maeda R 2015 Journal of Micromechanics and Microengineering 25 095017

[9] Wang D F, Chatani K, Ikehara T and Maeda R 2012 Microsystem technologies 18 1923-1929

[10] Thiruvenkatanathan P, Yan J, Woodhouse J, Aziz A and Seshia A 2010 Applied Physics Letters 96081913

[11] Manav M, Phani A S and Cretu E 2017 Journal of Micromechanics and Microengineering 27055010

[12] Humbert C, Goavec-Merou G, Walter V, Kacem N and Leblois T 2020 Smart Materials and Structures 29 02LT01

[13] Humbert C, Walter V, Kacem N and Leblois T 2020 Sensors 205295

[14] Zhao C, Wood G S, Xie J, Chang H, Pu S H and Kraft M 2016 Journal of Microelectromechanical Systems 25 626-636

[15] Juillard J, Prache P, Ferreira P M and Barniol N 2018 IEEE transactions on ultrasonics, ferroelectrics, and frequency control $652440-2448$

[16] Postma H C, Kozinsky I, Husain A and Roukes M 2005 Applied Physics Letters 86223105

[17] Rabenimanana T, Walter V, Kacem N, Le Moal P, Bourbon $\mathrm{G}$ and Lardiès J 2020 Applied Physics Letters 117 033502

[18] Ozdogan M, Daeichin M, Ramini A and Towfighian S 2017 Sensors and Actuators A: Physical 265 20-31

[19] Aryal N and Emadi A 2020 Micromachines 11401

[20] Pallay M and Towfighian S 2018 Applied Physics Letters 113213102

[21] Pallay M, Miles R N and Towfighian S 2021 Mechanical Systems and Signal Processing 150107250

[22] Hu F, Wang W and Yao J 2011 Journal of Micromechanics and Microengineering $\mathbf{2 1} 115029$

[23] He S and Mrad R B 2008 Journal of Microelectromechanical Systems 17 532-547

[24] Rabenimanana T, Walter V, Kacem N, Le Moal P, Bourbon G and Lardies J 2019 Sensors and Actuators A: Physical 295 643-652

[25] Walter V, Bourbon G, Le Moal P, Kacem N and Lardies J 2016 Procedia Engineering 168 1488-1491

[26] Cowen A, Hardy B, Mahadevan R and Wilcenski S 2011 Memscap Inc $\mathbf{1 3}$

[27] Kacem N, Arcamone J, Perez-Murano F and Hentz S 2010 Journal of Micromechanics and Microengineering 20 045023 